\title{
Energetische Nutzung von Fettabscheiderinhalten - Potenzial zur Erzeugung von Biodiesel und Biogas
}

\author{
Thomas Lichtmannegger · Carolina Kinzel (D) - Wolfgang Müller · Anke Bockreis
}

Online publiziert: 22. Januar 2018

(c) Der/die Autor(en) 2018. Dieser Artikel ist eine Open-Access-Publikation.

\begin{abstract}
Zusammenfassung Der Fettabscheider als Ressource bietet kostengünstige Rohstoffe für erneuerbare Energieerzeugung. Eine optimale Nutzung dieser Ressource kann durch die Trennung der Stofffraktionen in Öl und Speisereste erreicht werden. Während die Speisereste in der (Co-)Vergärung zur Erzeugung von Biogas genutzt werden können, kann das abgetrennte Öl als Substrat für die Biodieselherstellung dienen. Dieser Biodiesel aus Reststoffen stellt im Gegensatz zu Energiepflanzen keine Konkurrenz zur Nahrungsmittelerzeugung dar und trägt $\mathrm{zu}$ einer nachhaltigen und ressourcenschonenden Energieerzeugung bei. Durch die ungünstige Rohstoffqualität (hohe Konzentration freier Fettsäuren und Schwefel) sind moderne Biodiesel-Herstellungsprozesse notwendig. Die abgetrennten Speisereste mit ihrem erhöhten Restölgehalt erzielen in der Co-Fermentation immer noch hohe Biogaserträge, wodurch eine optimale energetische Nutzung möglich wird.
\end{abstract}

Schlüsselwörter Fettabscheider Erneuerbare Energie · Biodiesel . Biogas

\section{Energy recovery from grease traps wastes - potential for production of biodiesel and biogas}

\section{Abstract Grease traps as a resource provides low cost raw materials for re- newable energy production. Optimal use of this resource can be achieved by separating the material fractions into oil and food particles. While the \\ T. Lichtmannegger, MSc . \\ Dr. C. Kinzel $(\varangle) \cdot$ Dr.-Ing. W. Müller \\ Univ.-Prof. Dr.-Ing. A. Bockreis \\ Arbeitsbereich Umwelttechnik, \\ Institut für Infrastruktur \\ Universität Innsbruck, \\ Technikerstraße 13, 6020 Innsbruck, \\ Österreich \\ carolina.kinzel@uibk.ac.at}

residues can be used in the co-fermentation to produce biogas, the separated oil can serve as a substrate for biodiesel production. In contrast to energy crops, this biodiesel from waste material does not compete with food production and contributes to sustainable and resource-saving energy production. Due to the unfavourable raw material quality, (high concentration of free fatty acids and sulphur) modern biodiesel production processes are necessary. The separated food residues with increased residual oil content still produce high biogas yields in co-fermentation, which makes optimum energy utilization possible.

Keywords Grease traps · Renewable energy · Biodiesel · Biogas

\section{Einführung}

Fette im Abwasser verursachen Probleme bei der Abwasserentsorgung, unter anderem die gerade medial beachteten Fettberge im Kanalsystem. Aus diesem Grund versucht man in den meisten Ländern, ein geordnetes Fettmanagement $\mathrm{zu}$ etablieren (Wallace et al. 2017). In Österreich ist in der ÖNORM EN 1825-1:2005 festgelegt, welche Einrichtungen Fettabscheider brauchen. Jedoch ist es in der Praxis häufig so, dass Fettabscheider ganz fehlen oder $\mathrm{zu}$ selten gewartet werden und so ihre Funktionsfähigkeit eingeschränkt ist (Dengg und Rostek 2004; Hepperger und Hartmann 2011). Eine Möglichkeit die Situation zu verbessern, wäre die verstärkte Nutzung der Fettabscheiderinhalte als Ressource, umso die Installation und Wartung von Fettabscheidern attraktiver zu machen. Fettabscheiderinhalte können verschiedentlich verwertet werden, z. B. als Biogas durch (Co-)Vergärung, als Viehfutter oder als Ausgangssubstrat für Biodiesel (Montefrio et al. 2010). Die Erzeugung erneuerbarer Energie aus Fettabscheiderinhalten ist insofern eine interessante Option, da der
Anbau von Energiepflanzen in Konkurrenz zur Nahrungsmittelerzeugung steht und gezeigt werden konnte, dass die daraus erzeugte Energie eine höhere Kohlendioxidschuld produziert als fossile Energieträger (Fargione et al. 2008).

Aufgrund seiner großen Anfallmengen bieten Fettabscheiderinhalte ein großes Biogaspotenzial (Zhang et al. 2014). Die Co-Vergärung von Fettabfällen auf Kläranlagen steigert bis zu einer gewissen Zugabemenge den Biogasertrag deutlich, sowohl hinsichtlich der Menge als auch des Methananteils im Biogas (Davidsson et al. 2008; Del Mundo und Sutheerawattananonda 2017) und ist daher weit verbreitet (DWAM380 2009). Wang et al. (2013) konnten in ihrer Studie bei der Co-Vergärung Fettabscheiderinhalte bis $\mathrm{zu} 20 \%(\mathrm{v} / \mathrm{v})$ bzw. $65,5 \%(\mathrm{~m} / \mathrm{m})$ Trockensubstanz ohne Reaktorversagen zufüttern und dabei sowohl Biogasertrag als auch Methangehalt steigern.

Die Verwertung/Entsorgung von Fettabscheiderinhalten in Faultürmen kommunaler Kläranlagen ist in Österreich gängige Praxis. Fettabfälle haben hierbei ein sehr hohes Biogaspotenzial und die Gasqualität für die Verbrennung in Blockheizkraftwerken ist durch den hohen Anteil an Methan (ca. 68\%) sehr von Vorteil. Hierbei wird meist der gesamte Inhalt der Fettabscheider (fettreiche Schwimmschicht, wässrige Phase inklusive Spülwasser, Sediment) in einem beheizten Tank gelagert und kontinuierlich dem Faulturm zugegeben. Problematisch ist allerdings, dass der Wasseranteil in Fettabscheidern sehr hoch ist (oft bis zu 90\%). Diese wässrige Phase benötigt nicht nur Heizenergie während der Lagerung, sondern belegt auch Faulraum im Reaktor (Long et al. 2012).

Eine weitere erneuerbare Energiequelle ist Biodiesel. Problematisch mit Biodiesel aus Pflanzenölen sind neben der Nahrungsmittelkonkurrenz auch die hohen Rohstoffkosten, die diesen Biodiesel oft unwirtschaftlich machen 
(Canakci 2007; Math et al. 2010). In den letzten Jahren wurde jedoch eine Vielzahl verschiedener Verfahren zur Biodieselerzeugung entwickelt: homogen oder heterogen katalysierte Prozesse, einstufige und mehrstufige Verfahren, enzymatisch katalysierte Prozesse und überkritische Prozesse (BankovićIlić et al. 2014; Guldhe et al. 2015; Gumba et al. 2016). Dadurch ist heute die Nutzung verschiedenster Ausgangssubstrate möglich, auch minderwertige Abfallstoffe wie Klärschlamm (Mondala et al. 2009), tierische Abfallprodukte (Banković-Ilić et al. 2014) und Fettabscheiderinhalte (Wallace et al. 2017) können zur Biodieselerzeugung genutzt werden.

Getrennt erfasste Altspeiseöle sind aufgrund der geringen Rohstoffkosten zur Biodieselerzeugung wirtschaftlich interessant (Kulkarni und Dalai 2006) und es existieren verschiedene entsprechende Systeme wie in Tirol das Altspeiseöl-Sammelsystem Öli (Ortner et al. 2016).

Im Vergleich $\mathrm{zu}$ getrennt erfassten Altspeiseölen ist die Qualität der Altspeisefette und -öle (fat, oil and grease - FOG) aus Fettabscheidern deutlich schlechter. Durch Lipasen und Mikroorganismen in den Fettabscheidern kommt es zu einer Aufspaltung der Fette. Hierbei werden die Fettsäuren vom Triglycerid abgespalten und es kommt zu einer Zunahme an freien Fettsäuren (He und Yan 2016; Shon et al. 2002). Durch eine regelmäßige Leerung von Fettabscheidern in kurzen Intervallen ist es theoretisch möglich, ein Ausgangssubstrat mit einem relativ geringen Gehalt freier Fettsäuren zu erhalten (Montefrio et al. 2010).

Fette und Öle aus Fettabscheidern weisen im Vergleich zu separat gesammelten Altspeiseölen eine insgesamt schlechtere Qualität auf. Um die Eignung als Biodiesel zu erfüllen, ist die Cetanzahl ein wichtiger Richtwert. Dieser beschreibt die Zündwilligkeit des Kraftstoffs. Die minimale Cetanzahl für Biodiesel liegt laut ÖNORM EN 14214: 20170101 bei 51. Im Schnitt ist die Cetanzahl bei Biodiesel (Cetanzahl: 58) höher als bei mineralischem Dieselkraftstoff (Cetanzahl: 53 bis 54). Die Cetanzahl kann über das Fettsäureprofil (C14 bis C24) abgeschätzt werden. Vor allem mehrfach ungesättigte Fettsäuren wie zum Beispiel Linolensäure (C18:3) wirken sich negativ auf die Cetanzahl aus und verringern diese, wohingegen sich langkettige, gesättigte Fettsäuren positiv auf die Cetanzahl auswirken und diese erhöhen (Bamgboye und Hansen 2008).

Ein weiteres Problem bei der Biodieselproduktion aus Fettabscheider-FOG ist der erhöhte Schwefelgehalt (He et al. 2017; Hums et al. 2016). Vor allem Speisereste können hier zu einem starken Eintrag an Schwefel führen, da besonders in Lebensmitteln wie Eiern, Milchprodukten, Fleisch oder Lauchgewächsen viel Schwefel enthalten ist (Kemmerer und Boutwell 1932). Nach ÖNORM EN 14214 liegt der Grenzwert für Biodiesel bei $10 \mathrm{mg}$ Schwefel pro kg. Hums et al. (2016) hingegen setzen für ihre Ökobilanz bei Fettabscheidern einen mittleren Wert von 200 ppm Schwefel an.

Die Einhaltung des Grenzwerts kann im Herstellungsprozess durch mehrmalige Destillation erreicht werden. Sollte der Grenzwert nicht erreicht werden, muss ein zusätzliches Verfahren zur Entschwefelung nachgeschaltet werden (Reichel und Raudner 2016).

Für die industrielle Erzeugung von Biodiesel ist vor allem der Parameter Freie Fettsäuren (FFS) die zentrale Größe. Dieser Parameter bestimmt maßgeblich, welches Verfahren großtechnisch eingesetzt wird, um das Öl in Biodiesel umzuwandeln. Liegen die FFS $<5 \%$, ist das alkalisch-katalytische Verfahren die wirtschaftlichste Variante. Für Qualitäten mit FFS $>5 \%$ werden sogenannte Multi-Feedstock-Verfahren (BDI-BioEnergy International AG) angeboten, die einen Gehalt an FFS von bis $\mathrm{zu} 100 \%$ erlauben. Die Biodieselherstellung aus Fettabscheider-FOG braucht aufgrund des hohen Gehalts freier Fettsäuren und Wasser andere Verfahren als das Standardverfahren der basisch katalysierten Umesterung. Hums et al. (2016) zeigten jedoch, dass die Ökobilanz von Biodiesel aus Fettabscheider-FOG vergleichbar gut wie die etablierter Treibstoffe sein kann. In der Ökobilanz waren bei Fettgehalten der Fettabscheiderinhalte über $10 \%$ die meisten der untersuchten UmweltMaßzahlen geringer als die von schwefelarmem Treibstoff und vergleichbar mit Sojabohnen-Biodiesel.

In der vorliegenden Arbeit soll aufgezeigt werden, wie Fettabscheiderinhalte energetisch möglichst hochwertig genutzt werden können. Es wurden Proben aus Fettabscheidern unterschiedlichen Zustands gezogen und die in der Schwimmschicht enthaltenen Altspeisefette und -öle hinsichtlich ihrer Qualität (Gehalt freier Fettsäuren, Schwefel und Fettsäurezusammensetzung) analysiert. Das Biogaspotenzial von Fettabscheiderinhalten wurde für die einzelnen enthaltenen Fraktionen getrennt bestimmt. Die Untersuchungen zeigen das Potenzial von Fettabscheiderinhalten für die Biodieselherstellung, auch wenn ihre Verwertung aufgrund der im Vergleich zu getrennt erfassten Altspeiseölen geringeren Qualität aufwendigere Verfahren erfordert. Die bei der Aufarbeitung der Fettabscheiderinhalte anfallenden Reststoffe (Speisereste) weisen ein hohes Gasbildungspotenzial auf und sind weiterhin für die energetische Nutzung in der (Co-)Vergärung interessant.

\section{Material und Methoden}

\subsection{Probennahme}

Für die Untersuchungen wurden elf Fettabscheider beprobt, bei welchen das Datum der letzten Entleerung und Wartung durch lokale Entsorger bekannt war. Es wurden Proben aus der Schwimmschicht der Fettabscheider genommen, um den quantitativen Anteil an Fett und Speiseresten zu messen und die Fettqualität in Hinsicht auf die Biodieselherstellung zu bestimmen. Des Weiteren wurde aus dem Ablauf des Fettabscheiders eine Abwasserprobe gezogen. Bei der Beprobung wurde darauf geachtet, möglichst wenig Turbulenzen im Ablauf zu erzeugen, um eine Rücklösung von Fett zu vermeiden. Zusätzlich wurden grobe Fettpartikel mit einem Sieb $(250 \mu \mathrm{m})$ abgeschieden. In den Ablaufproben wurde der $\mathrm{pH}$-Wert gemessen und die schwerflüchtigen lipophilen Stoffe nach dem gravimetrischen Verfahren DIN ISO 11349:201512 bestimmt.

\subsection{Laboranalysen}

Für die Messung der freien Fettsäuren/ Säurezahl wurden die gefilterten Fettproben auf $60^{\circ} \mathrm{C}$ erhitzt und mit einem Metrohm Titrando 888 nach DIN EN ISO 660:2009-10 untersucht. Die Ergebnisse werden als Prozent an freien Fettsäuren (\%) dargestellt.

Für die Analyse des Fettsäurespektrums wurden die Fettproben mit TMSH zu Fettsäuremethylestern (FAME) verestert (DGF-Einheitsmethoden C-VI 11e (98) (2016)) und im Anschluss mit einem Gaschromatograph (Shimadzu GC2010 plus mit Macherey Nagel Opti- 
ma Biodiesel F Säule) gemessen (DGFEinheitsmethoden C-VI 10a (2016)).

Die Schwefelmessung wurde von der Firma ASG Analytik durchgeführt (DIN EN ISO 20884:2011-07).

\subsection{Gasbildungspotenzial von Fettabscheiderinhalten im Gärtest $\left(\mathrm{GB}_{21}\right)$}

Der Gärtest erfolgte nach VDI 4630: 2016-11, die Gasbildung wurde mit einem automatischen MethanpotenzialTest-System (AMPTS) der Firma Bioprocess Control bestimmt. Aus dem Fettabscheider eines Restaurants mit ca. 550 Essen pro Tag wurden Proben der fetthaltigen Schwimmschicht und der Flüssigphase genommen. Die homogenisierte Probe wurde zentrifugiert, um das Speisereste-Öl-Gemisch in der Schwimmschicht effektiv $\mathrm{zu}$ trennen. Im Anschluss wurde das Gaspotenzial der gesamten Schwimmschichtprobe (Speisereste + Öl) und der Einzelkomponenten (Speisereste, Öl, wässrige Phase) bestimmt.

\subsection{Biodieselqualität}

Anhand der Fettsäuremuster kann man die zu erwartende Cetanzahl mit nachfolgender Formel (Gl. 1) berechnen (Bamgboye und Hansen 2008):

$$
\begin{array}{r}
\mathrm{CN}=61,1+0,088 x_{2}+0,133 x_{3}+0,152 x_{4} \\
-0,101 x_{5}-0,039 x_{6}-0,243 x_{7}-0,395 x_{8}
\end{array}
$$

$\mathrm{x}_{2}$ bis $\mathrm{x}_{8}$ sind die prozentuale Zusammensetzung der gemessenen Fettsäuren ( $\mathrm{x}_{2}$ Myritinsäure (C14:0); $\mathrm{x}_{3}$ Palminsäure (C16:0); $\mathrm{x}_{4}$ Stearinsäure (C18:0); $\mathrm{x}_{5}$ Palmitoleinsäure (C16:1); $\mathrm{x}_{6}$ Ölsäure (C18:1); $x_{7}$ Linolsäure (C18:2); $\mathrm{x}_{8}$ Linolensäure (C18:3)).

\section{Ergebnisse}

\subsection{Zustandsbeurteilung und Ablaufwerte der Fettabscheider}

Im Rahmen der Beprobung der Fettabscheider wurde auch deren Betriebszustand beurteilt. Die letzte Entleerung/ Wartung der Abscheider lag zwischen 20 und 355 Tagen zurück. Als Parameter für die Beurteilung dienten sowohl die Schwimmschicht (Dicke und Ausprägung) als auch das Vorhandensein von Fett im Bereich des Ablaufs. Die Ergebnisse sind in Tab. 1 dargestellt. In vier der elf Fettabscheider war noch

Tab. 1 Ablaufmessung und Zustandsbeurteilung der Fettabscheider. Zustandsbeurteilung: gut: keine geschlossene Schwimmschicht oder Schicht $<5 \mathrm{~cm}$, kein Fett im Ablauf; mangelhaft: geschlossene Schwimmschicht oder Schicht $>10 \mathrm{~cm}$

\begin{tabular}{|c|c|c|c|c|}
\hline & \multicolumn{3}{|c|}{ Ablauf Fettabscheider } & \multirow[t]{2}{*}{ Zustandsbeurteilung } \\
\hline & Alter $[\mathrm{t}]$ & pH Wert & Lipo.Stoffe [mg/L] & \\
\hline FA1 & 125 & 9,6 & 462 & Mangelhaft \\
\hline FA2 & 100 & 4,8 & 409 & Mangelhaft \\
\hline FA3 & 43 & 4,5 & 124 & Gut \\
\hline FA4 & 189 & 4,4 & 185 & Gut \\
\hline FA5 & 38 & 5,1 & 93 & Mangelhaft \\
\hline$F A 6^{a}$ & 159 & 6,1 & 266 & Gut \\
\hline$F A T^{b}$ & 103 & - & - & Mangelhaft \\
\hline FA8 & 20 & 5,1 & 234 & Gut \\
\hline FA9 & 123 & 5,5 & 123 & Gut \\
\hline$F A 10^{C}$ & 76 & 5,1 & 453 & - \\
\hline FA11 & 355 & 6,07 & 109,1 & Mangelhaft \\
\hline
\end{tabular}
mit Fettaustrag im Ablauf

keine geschlossene Schwimmschicht ausgebildet und kein Fett im Auslauf ersichtlich. Einer der untersuchten Abscheider war ein sogenannter „Direktfettabscheider“, bei dem die Speisereste im Zulauf durch ein Sieb abgetrennt, das Fett täglich automatisch abgezogen und in separaten Fässern gelagert wurde. Fünf der Fettabscheider hatten eine geschlossene, deutlich ausgeprägte Schwimmschicht im Abscheideraum und teilweise beträchtliche Fettablagerungen im Bereich des Ablaufs (Fettaustrag). Einer der untersuchten Abscheider (FA10) war nicht funktionsfähig, da keine Trennwände und Tauchrohre vorhanden waren, wodurch eine Abscheidung von Fetten nicht möglich war. Insgesamt waren fünf Abscheider gut gewartet und voll funktionsfähig. Bei fünf Abscheidern war eine Entleerung und Wartung nötig, um die Abscheideleistung wiederherzustellen (Abb. 1), ein Abscheider bedurfte einer Generalsanierung.

Die Entnahme einer repräsentativen Ablaufprobe gestaltet sich in der Praxis schwierig. Vor allem Fettablagerungen im Ablaufbereich, die sich bei der Beprobung loslösen können, oder mangelnde Beschickung des Fettabscheiders außerhalb der Stoßzeiten, können zu einer starken Verschiebung der Messwerte führen.

Für die Parameter pH-Wert und schwerflüchtige lipophile Stoffe gibt es laut Abwasseremissionsverordnung (BGBl. Nr. 186/1996) Grenzwerte für die Einleitung in das öffentliche Kanalnetz. Diese Verordnung schreibt einen $\mathrm{pH}$ -
Wert zwischen $\mathrm{pH}$ 6,5 und $\mathrm{pH}$ 9,5 vor. Neun Ablaufproben liegen unter dem geforderten Wert, eine Probe liegt darüber. Generell ist zu vermerken, dass das Vorhandensein von freien Fettsäuren den $\mathrm{pH}$-Wert erniedrigt und so Richtung saures Milieu verschiebt. Dadurch ist die Einhaltung des Grenzwertes stark erschwert und durch den Einsatz von sauren Reinigungsmitteln kann dieser Umstand noch weiter verstärkt werden. Der gemessene $\mathrm{pH}$-Wert von $\mathrm{pH}$ 9,6 in Fettabscheider 1 ist auf den Einsatz von basischen Reinigungsmitteln zurückzuführen, die man bei der Verarbeitung der Probe deutlich am Geruch feststellen konnte.

Für die schwerflüchtigen lipophilen Stoffe schreibt das BGBl. Nr. 186/1996 einen Grenzwert von $100 \mathrm{mg} / \mathrm{L}$ vor. Von zehn gemessenen Proben konnte lediglich eine den geforderten Grenzwert einhalten und in drei Fällen wurde er sogar um das Vierfache überschritten.

Anhand der Erhebungen ist kein direkter Zusammenhang zwischen der letzten Entleerung und dem Betriebszustand erkennbar.

\subsection{Fettqualität der Fettabscheider- FOG}

\subsubsection{Freie Fettsäuren}

Für die Nutzung der Fettabscheider FOG für die Biodieselherstellung ist der Parameter freie Fettsäuren (FFS) ausschlaggebend. Der Gehalt an freien Fettsäuren in den beprobten Fettabscheidern war bei allen untersuchten 


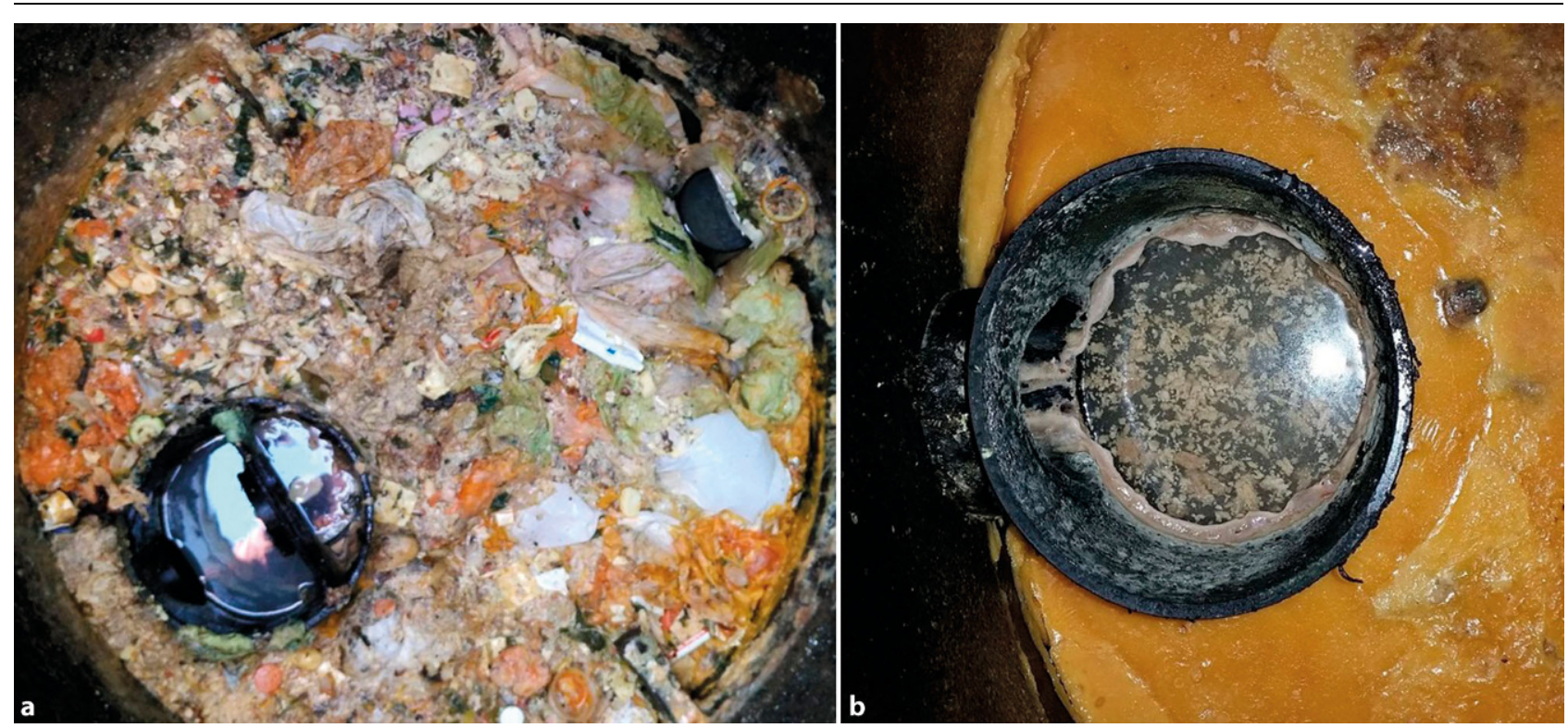

Abb. 1 a Schwimmschicht eines Fettabscheiders bestehend aus Speiseresten und Abfall-Fehlwürfen; $\mathbf{b}$ voller Fettabscheider mit Fettablagerungen im Ablaufbereich

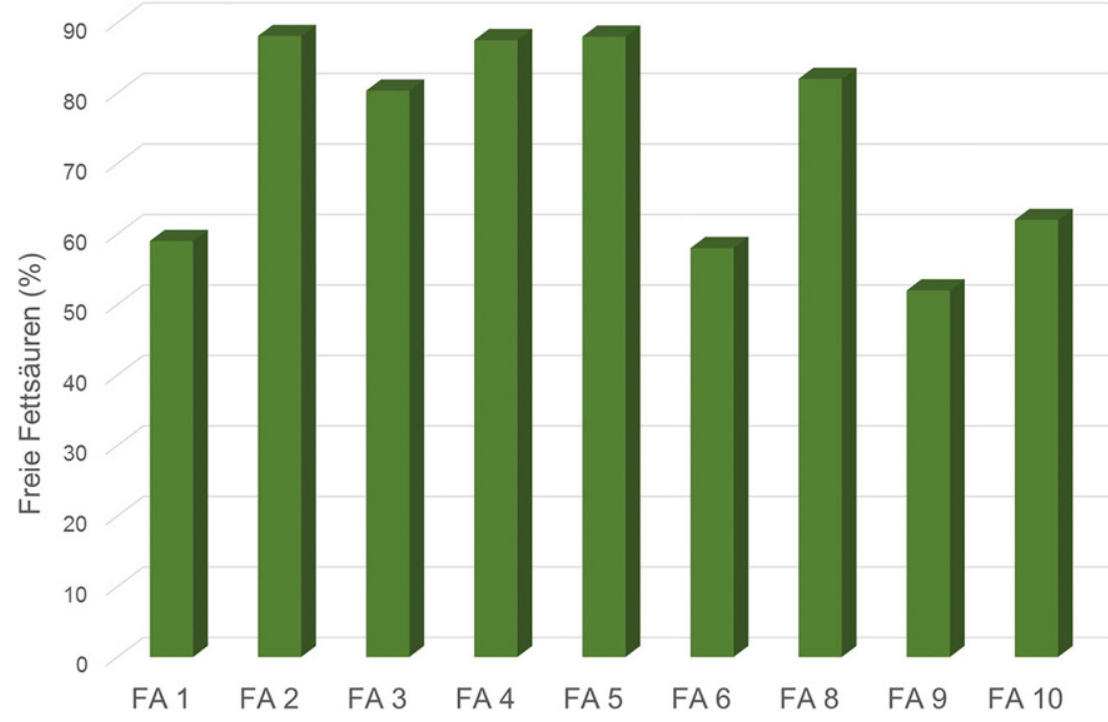

Abb. 2 Gehalt an freien Fettsäuren (FFS) in den beprobten Fettabscheidern (FA)

Fettabscheidern hoch, im Median $80 \%$ freie Fettsäuren (52 bis $88 \%$ ). Die Verteilung ist in Abb. 2 dargestellt. Es konnte kein direkter Zusammenhang zwischen dem Zeitraum seit der letzten Leerung des Fettabscheiders und dem FFS-Gehalt gefunden werden. Tendenziell waren in den Fettabscheidern mit den geringeren FFS-Konzentration weniger Speisereste in der Schwimmschicht.

\subsubsection{Schwefelgehalt}

Ein weiterer wichtiger Parameter für die Biodieselherstellung ist der Schwefel- gehalt aus den Fettabscheider-FOG. In Abb. 3 sind die Ergebnisse der Schwefelmessungen der Fettabscheiderproben dargestellt.

Bei den untersuchten Fettabscheidern lagen die Werte zwischen 31 und $519 \mathrm{mg} / \mathrm{kg}$. Drei Fettabscheider (FA1, FA3, FA6) zeigten sehr niedrige Schwefelkonzentrationen. Zieht man hierbei die Erkenntnisse der Probenahme in Hinsicht auf den Zustand des Fettabscheiders heran, so zeigt sich, dass alle diese Fettabscheider nur einen geringen Anteil an Speiseresten aufwiesen. Die Fettabscheider FA2, FA4, FA5 und
FA7 hingegen hatten einen hohen Anteil an Speiseresten in der Fettschicht.

\subsubsection{Cetanzahl}

Die zu erwartende Cetanzahl kann anhand der Fettsäuren im Ausgangssubstrat abgeschätzt werden. Dafür wird von den Fetten das Fettsäureprofil bestimmt. In Abb. 4 sind die Anteile der enthaltenen Fettsäuren in den beprobten Fettabscheidern dargestellt.

Die zu erwartende Cetanzahl des Biodiesels wurde mithilfe der von Bamgboye und Hansen (2008) entwickelten Formel (Gl. 1) berechnet. Die so erhaltenen Cetanzahlen liegen zwischen 52 und 63 in den gemessenen Fettabscheiderproben (Median 57) und erfüllen somit die Anforderungen für Biodiesel.

\subsection{Gasbildungspotenzial von Fettabscheiderinhalten}

Die Co-Vergärung von Fettabscheiderinhalten ist in Österreich gängige Praxis. Um das Gasbildungspotenzial der einzelnen Bestandteile zu beurteilen, wurde der $\mathrm{GB}_{21}$ der verschiedenen Fraktionen bestimmt. Die Biogaserträge der einzelnen Fraktionen sind in Abb. 5 dargestellt.

Den größten Biogasertrag liefert hierbei das Öl mit $1227 \mathrm{NL} / \mathrm{kg}$ oTS $( \pm 8)$, gefolgt von Speisereste + Öl mit $1126 \mathrm{NL} / \mathrm{kg}$ oTS $( \pm 8)$ und den abgetrennten Speiseresten mit $1017 \mathrm{NL} / \mathrm{kg}$ 


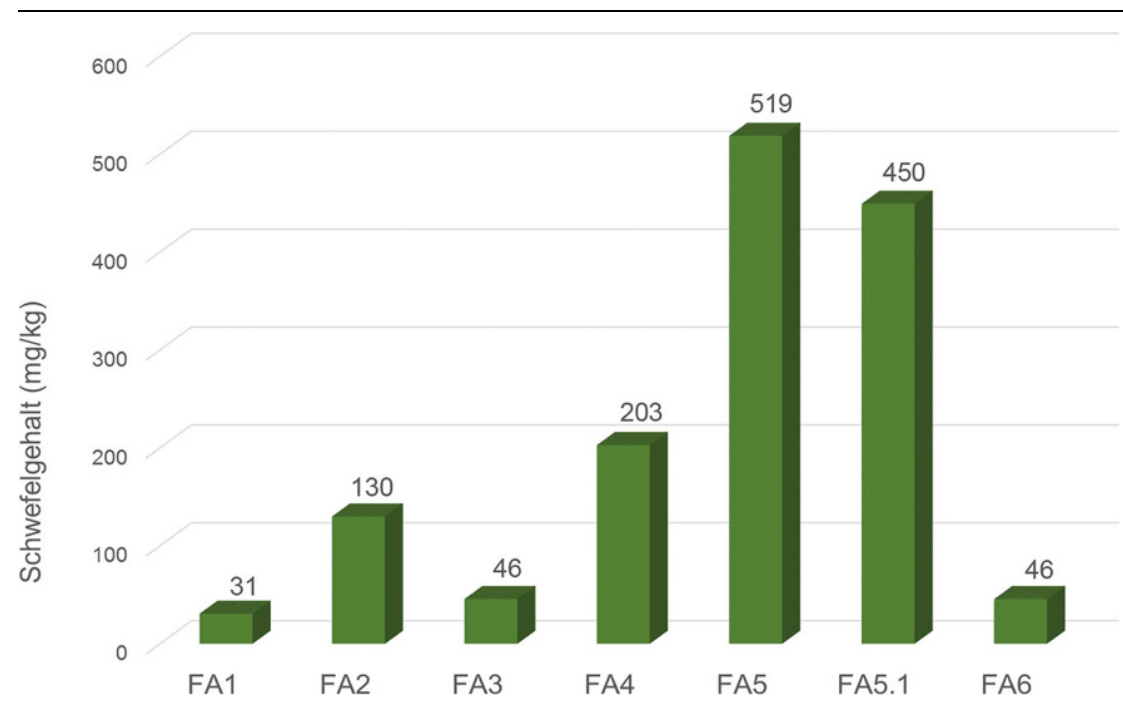

Abb. 3 Schwefelgehalt in Fetten aus Fettabscheidern (FA)

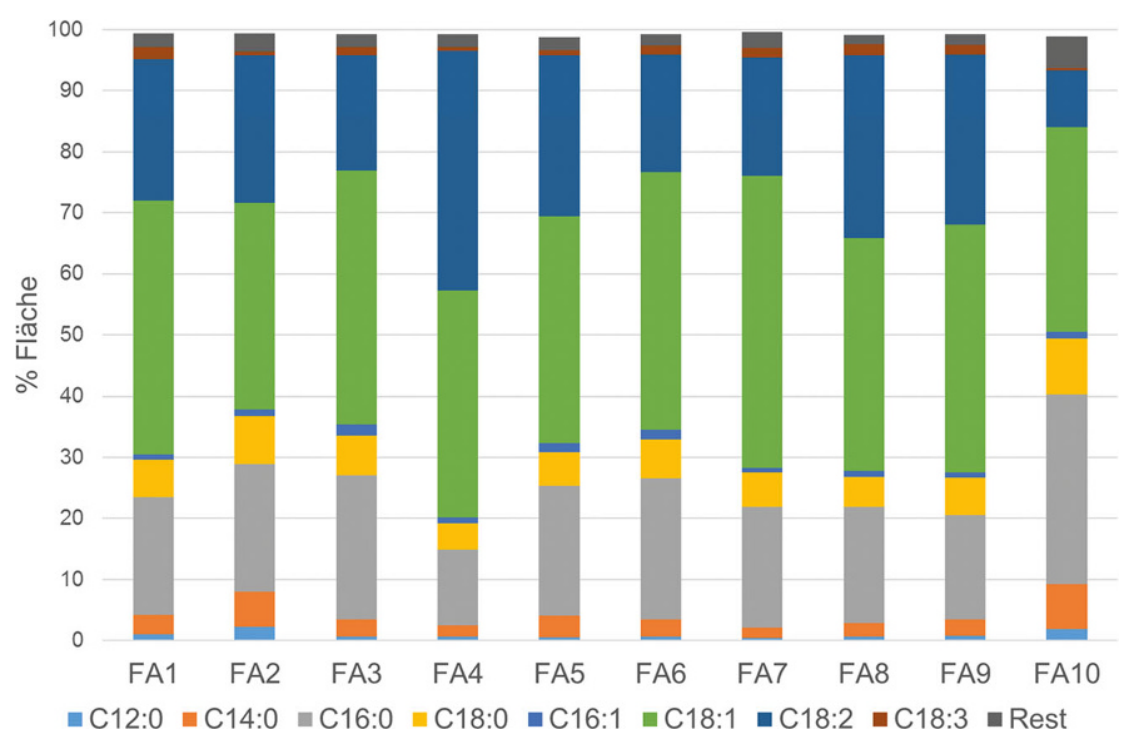

Abb. 4 Verteilung der Fettsäuren (C12 bis C24) in den Fettabscheider-Proben

$( \pm 17)$ oTS. Die wässrige Phase hingegen liefert kaum Biogas und ist mit $73 \mathrm{NL} / \mathrm{kg}$ oTS $( \pm 39)$ für die Biogaserzeugung kaum interessant.

Vergleicht man hier jedoch den Biogasertrag der Speisereste mit Öl und der entölten Speisereste, so ergibt sich hier lediglich eine Differenz von 109 NL/kg oTS. Der Ertrag der entölten Speisereste-Fraktion ist also lediglich ca. $10 \%$ geringer, wenn man das Öl aus den Fettabscheiderabfällen entfernt. Ein Grund für den hohen Biogasertrag der entölten Speisereste ist der immer noch hohe Anteil an Lipiden (Restölgehalt 18\%).

\section{Diskussion}

Die Beprobung zeigte, dass bei den meisten Fettabscheidern viel Potenzial zur Optimierung der Wartungs- und Entleerungsintervalle besteht. Durch mangelnde Entleerungen ist die Funktionsfähigkeit oft eingeschränkt (z. B. zu dicke Fettschicht, zu viele Speisereste). Dies wirkt sich nicht nur auf die Qualität der Fettabscheider-FOG aus, sondern bedeutet auch einen erheblichen Austrag an verwertbaren Ressourcen aus den Fettabscheidern. So wurde in den in der Schwimmschicht der Fettabscheider enthaltenen Altspeisefetten und -ölen ein hoher Anteil freier Fettsäuren (FFS) gefunden. Der Gehalt an FFS lag zwischen 52 und $88 \%$ (Median
$80 \%$ ). Eine alkalisch-katalysierte Umesterung zu Biodiesel kommt für diese Öle daher nicht in Frage. Für dieses Substrat muss daher ein mehrstufiges Verfahren angewendet werden, um eine wirtschaftlich sinnvolle Biodieselherstellung $\mathrm{zu}$ erreichen. Je mehr Verfahrensschritte benötigt werden, umso kostenintensiver wird die Herstellung des Biodiesels. Dieser Nachteil wird jedoch durch den günstigen Substratpreis wieder relativiert. Die FOG der untersuchten Fettabscheider enthielten im Median $130 \mathrm{mg} / \mathrm{kg}$ Schwefel (31 bis $519 \mathrm{mg}$ ) und liegen so in einem ähnlichen Bereich wie in der Literatur angegeben. Das bedeutet, dass der Biodiesel aus Fettabscheider-FOG entschwefelt werden muss, um den vorgeschriebenen Grenzwert von $10 \mathrm{mg} / \mathrm{kg}$ in Biodiesel zu erreichen. FOG aus Fettabscheidern mit einem hohen Anteil an Speiseresten wiesen tendenziell höhere Schwefelgehalte auf. Eine Abtrennung der Speisereste vor dem Eintrag in den Fettabscheider ist daher sinnvoll und kann zum Beispiel durch eine Schulung des Gastronomiepersonals oder angepasste Vorreinigung des Geschirrs erreicht werden. Durch diese einfachen Maßnahmen könnte so die Fettqualität für die Biodieselproduktion deutlich gesteigert und zusätzlich die Schwefelwasserstoff-Problematik im Kanalnetz (Geruchsbildung, Korrosion) reduziert werden.

Damit Öle und Fette für die Biodieselherstellung verwendet werden können, müssen sie eine minimale Cetanzahl von 51 aufweisen (ÖNORM EN 14214). Berechnet wird diese aus der Zusammensetzung einiger Fettsäuren wie in Gl. 1 dargestellt. Hierfür ist das Fettsäureprofil der Öle zu bestimmen. Anhand dieser Berechnungen zeigt sich, dass der Median der Cetanzahl bei 57 liegt und somit alle untersuchten Fettabscheiderproben für die Biodieselherstellung geeignet wären.

Da der Fettverderb bei konstantem Nährstoffeintrag und adaptierten Mikroorganismen sehr schnell vor sich geht, ist die Fettqualität aus Fettabscheidern für die Biodieselherstellung sehr schlecht bezogen auf den Anteil an FFS. Ein optimiertes Entsorgungsintervall bietet hier nur wenig Spielraum für eine Verbesserung der Fettqualität hinsichtlich FFS, jedoch könnten sich dadurch die Mengen an abgeschiedenem Fett erhöhen und der Fetteintrag in den Kanal maßgeblich verringert werden. Eine verbesserte Abtrennung 


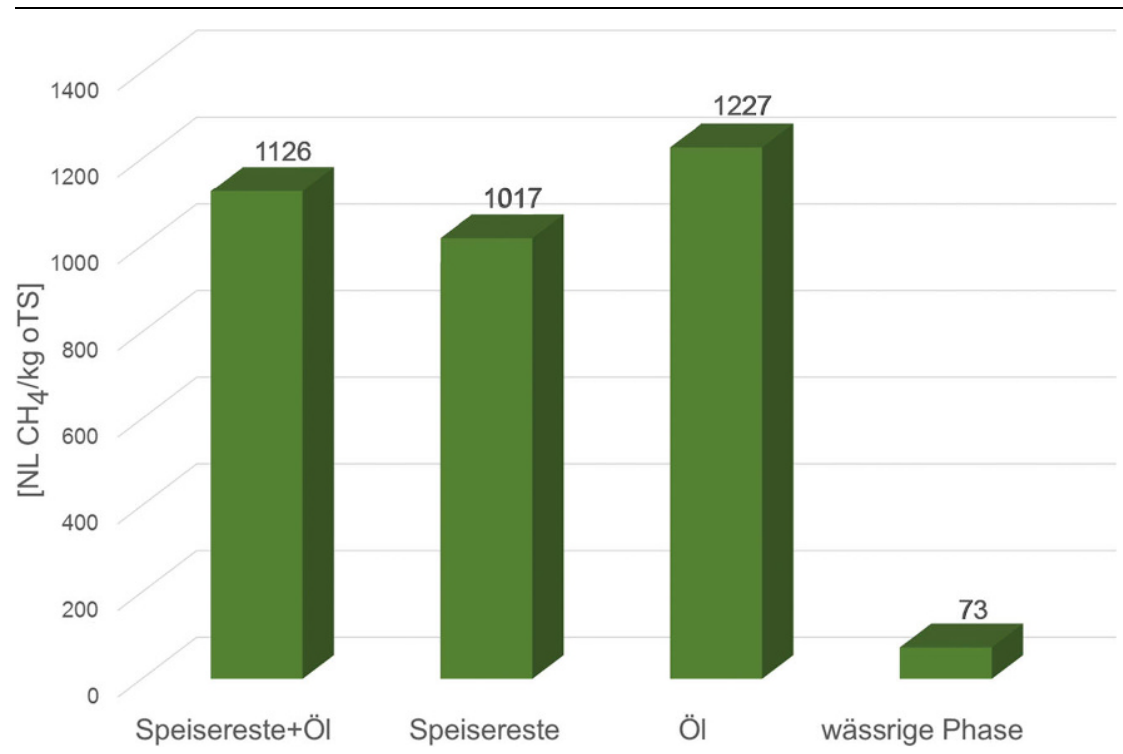

Abb. 5 Gasbildungspotenzial der getrennten Fraktionen: Speisereste + Öl, entölte Speisereste, Öl und wässrige Phase

von Speiseresten ist hier eine sinnvolle Maßnahme, um zum einen die Fettqualität zu verbessern und zum anderen den Fettabscheider effizienter zu nutzen, da nur Fette und keine Speisereste aus dem Abwasser abgeschieden werden müssen.

Um die Fettabscheiderinhalte möglichst hochwertig zu verwerten und die maximale Energierückgewinnung $\mathrm{zu}$ erhalten, ist eine Trennung der Stofffraktionen sinnvoll. Hierbei wird der gesamte Fettabscheiderinhalt in Fest-, Flüssig- und Ölphase über Zentrifugation bei $80^{\circ} \mathrm{C}$ getrennt. Für groß- dieses Rohstoffes kann erreicht werden, wenn die Wartungsintervalle verkürzt und eine saubere Trennung von Speiseresten und fetthaltigen Abwässern in der Küche erfolgt. Eine energetisch möglichst hochwertige Nutzung kann durch eine getrennte Verwertung der einzelnen Fraktionen erreicht werden, Fette zu Biodiesel und Speisereste in die Co-Vergärung.

Danksagung Das Projekt „BioPot Bioenergie aus Speisefetten - Potential gemischt erfasster Fettabfälle als Ressource für Biodiesel“ wird im Rahmen des Klima- und Energiefonds der FFG im Programm Energieforschung (e!MISSION), Energieforschung 2. Ausschreibung gefördert.

Funding Open access funding provided by University of Innsbruck and Medical University of Innsbruck.

Open Access Dieser Artikel wird unter der Creative Commons Namensnennung 4.0 International Lizenz (http:// creativecommons.org/licenses/by/4. $0 /$ deed.de) veröffentlicht, welche die Nutzung, Vervielfältigung, Bearbeitung, Verbreitung und Wiedergabe in jeglichem Medium und Format erlaubt, sofern Sie den/die ursprünglichen Autor(en) und die Quelle ordnungsgemäß nennen, einen Link zur Creative Commons Lizenz beifügen und angeben, ob Änderungen vorgenommen wurden.

Literatur

Bamgboye, A.I., Hansen, A.C. (2008): Prediction of cetane number of biodiesel fuel from the fatty acid methyl ester (FAME) composition. International Agrophysics 22 (1):21-29

Banković-Ilić, I.B., Stojković, I.J., Stamenković, O.S., Veljkovic, V.B., Hung, Y.-T. (2014): Waste animal fats as feedstocks for biodiesel production. Renewable and Sustainable Energy Reviews 32:238-254. https://doi.org/10.1016/j.rser.2014. 01.038

BGBl. Nr. 186/1996 (1996): Verordnung des Bundesministers für Land- und Forstwirtschaft über die allgemeine Begrenzung von Abwasseremissionen in Fließgewässer und öffentliche Kanalisationen (AAEV). Oesterreichisches Bundesgesetzblatt, Verordnung 186 BGBl. Nr. 186/1996 Canakci, M. (2007): The potential of restaurant waste lipids as biodiesel feedstocks. Bioresource Technology 98 (1):183-190. https://doi.org/10. 1016/j.biortech.2005.11.022

Davidsson, A., Lövstedt, C., la Cour Jansen, J., Gruvberger, C., Aspegren, H. (2008): Co-digestion of grease trap sludge and sewage sludge. Waste Management 28:986-992. https://doi.org/10. 1016/j.wasman.2007.03.024
Dengg, J., Rostek, R. (2004): Fett im Abwasser - Broschüre zur Reduzierung des Fetteintrags in das Abwasser. Abwasserverband AchentalInntal-Zillertal, Österreich.

DGF-Einheitsmethoden C-VI 10a (2016): Gaschromatographie: Analyse der Fettsäuren und Fettsäureverteilung. In: Deutsche Einheitsmethoden zur Untersuchung von Fetten, Fettprodukten, Tensiden und verwandten Stoffen. Wissenschaftliche Verlagsgesellschaft $\mathrm{GmbH}$, Stuttgart

DGF-Einheitsmethoden C-VI 11e (98) (2016): Fettsäuremethylester (TMSH Methode). In Deutsche Einheitsmethoden zur Untersuchung von Fetten, Fettprodukten, Tensiden und verwandten Stoffen. Wissenschaftliche Verlagsgesellschaft GmbH, Stuttgart

DIN EN ISO 20884:2011-07 (2011): Mineralölerzeugnisse - Bestimmung des Schwefelgehaltes in Kraftstoffen für Kraftfahrzeuge - Wellenlängendispersive Röntgenfluoreszenz-Spektrometrie (ISO 20884:2011); Deutsche Fassung EN ISO 20884:2011.

DIN EN ISO 660:2009-10 (2009): Tierische und pflanzliche Fette und Öle - Bestimmung der Säurezahl und der Azidität (ISO 660:2009).
DIN ISO 11349:2015-12 Wasserbeschaffenheit - Bestimmung von schwerflüchtigen lipophilen Stoffen - Gravimetrisches Verfahren (ISO 11349:2010).

DWA-M380 (2009), Co-Vergärung in kommunalen Klärschlammfaulbehältern, Abfallvergärungsanlagen und landwirtschaftlichen Biogasanlagen. Deutsche Vereinigung für Wasserwirtschaft, Abwasser und Abfall e.V. ISBN:978-3941089-63-1

Fargione, J., Hill, J., Tilman, D., Polasky, S., Hawthorne, P. (2008) Land Clearing and the Biofuel Carbon Debt. Science 319 (5867):1235$1238 . \quad$ https://doi.org/10.1126/science. 1152747

Guldhe, A., Singh, B., Mutanda, T., Permaul, K., Bux, F. (2015): Advances in synthesis of biodiesel via enzyme catalysis: Novel and sustainable approaches. Renewable and Sustainable Energy Reviews 41:1447-1464. https://doi.org/10.1016/ j.rser.2014.09.035

Gumba, R.E., Saallah, S., Misson, M., Ongkudon, C.M., Anton, A. (2016): Green biodiesel production: a review on feedstock, catalyst, monolithic reactor, and supercritical fluid tech- 
nology. Biofuel Research Journal 3 (3):431-447. https:// doi.org/10.18331/brj2016.3.3.3

He, X., Yan, T. (2016): Impact of microbial activities and hydraulic retention time on the production and profile of long chain fatty acids in grease interceptors: a laboratory study. Environ SciWat Res Technol 2 (3):474-482. https://doi.org/ 10.1039/c6ew00013d

He, X., de los Reyes, F.L., Ducoste, J.J. (2017): A critical review of fat, oil and grease (FOG) in sewer collection systems: challenges and control Critical Reviews in Environmental Science and Technology. https://doi.org/10.1080/10643389. 2017.1382282

Hepperger, O., Hartmann, S. (2011): Neue Wege zur Fettreduktion im Abwasser am Beispiel der Talschaft Montafon. Bericht des Abwasserverbands Montafon Vandans, Österreich

Hums, M. E., Cairncross, R.A., Spatari, S. (2016): Life-Cycle Assessment of Biodiesel Produced from Grease Trap Waste. Environmental Science \& Technology 50 (5):2718-2726. https://doi.org/ 10.1021/acs.est.5b02667

Kemmerer, K., Boutwell, P. (1932): Sulfur content of foods. Industrial \& Engineering Chemistry Analytical Edition 4 (4):423-425

Kulkarni, M.G., Dalai, A.K. (2006): Waste cooking oil - An economical source for biodiesel: A review Industrial and Engineering Chemistry Research 45 (9):2901-2913. https://doi.org/10 $1021 / \mathrm{ie} 0510526$

Long, J.H., Aziz, T.N., Reyes, F.Ldl, Ducoste, J.J. (2012): Anaerobic co-digestion of fat, oil, and grease (FOG): A review of gas production and process limitations. Process Safety and Environ- mental Protection 90 (3):231-245, https://doi. org/10.1016/j.psep.2011.10.001

Math, M.C., Kumar, S.P., Chetty, S.V. (2010): Technologies for biodiesel production from used cooking oil - A review. Energy for Sustainable Development 14 (4):339-345. https://doi.org/10 1016/j.esd.2010.08.001

Mondala, A., Liang, K., Toghiani, H., Hernandez, R., French, T. (2009): Biodiesel production by in situ transesterification of municipal primary and secondary sludges. Bioresource Technology 100 (3):1203-1210. https://doi.org/10. 1016/j.biortech.2008.08.020

Montefrio, M.J., Xinwen, T., Obbard, J.P. (2010): Recovery and pre-treatment of fats, oil and grease from grease interceptors for biodiesel production. Applied Energy 87 (10):3155-3161. https:// doi.org/10.1016/j.apenergy.2010.04.011

Del Mundo, D.M.N., Sutheerawattananonda, M. (2017): Influence of fat and oil type on the yield, physico-chemical properties, and microstructure of fat, oil, and grease (FOG) deposits. Water Research 124 (Supplement C):308-319. https://doi.org/10.1016/j.watres. 2017.07.047

ÖNORM EN 14214: 20170101 (2017): Flüssige Mineralölerzeugnisse - Fettsäure-Methylester (FAME) zur Verwendung in Dieselmotoren und als Heizöl - Anforderungen und Prüfverfahren (konsolidierte Fassung).

ÖNORM EN 1825-1: 20050101 (2005): Abscheideranlagen für Fette - Teil 1: Bau-, Funktionsund Prüfgrundsätze, Kennzeichnung und Güteüberwachung
Ortner, M.E., Müller, W., Schneider, I., Bockreis, A. (2016): Environmental assessment of three different utilization paths of waste cooking oil from households. Resources, Conservation and Recycling 106:59-67. https://doi.org/10. 1016/j.resconrec.2015.11.007

Reichel, M., Raudner, R. (2016): Verfahren zur Reinigung von Fettsäurealkylestern. WO20160 $91640 \mathrm{Al}, 2016$

Shon, H.K., Tian, D., Kwon, D.Y., Jin, C.S., Lee, T.J., Chung, W.J. (2002): Degradation of fat, oil, and grease (FOGs) by lipase-producing bacterium Pseudomonas sp strain D2D3. J Microbiol Biotechnol 12 (4):583-591

VDI 4630:2016-11 (2016): Vergärung organischer Stoffe - Substratcharakterisierung, Probenahme, Stoffdatenerhebung, Gärversuche

Wallace, T., Gibbons, D., O'Dwyer, M., Curran, T.P. (2017): International evolution of fat, oil and grease (FOG) waste management - A review. J Environ Manage 187:424-435. https://doi.org/ 10.1016/j.jenvman.2016.11.003

Wang, L., Aziz, T.N., de los Reyes, F.L. (2013): Determining the limits of anaerobic co-digestion of thickened waste activated sludge with grease interceptor waste. Water Reaserch 47:3835-3844. https://doi.org/10.1016/j.watres.2013.04.003 Zhang, P., Lin, C.-J., Liu, J., Pongprueksa, P., Evers, S.A., Hart, P. (2014): Biogas production from brown grease using a pilot-scale highrate anaerobic digester. Renewable Energy 68:304-313. https://doi.org/10.1016/j.renene. 2014.01.046 